(2) Open Access Full Text Article

\title{
Aneuploid Circulating Tumor Cells as a Predictor of Response to Neoadjuvant Chemotherapy in Non-Small Cell Lung Cancer
}

\author{
Miao Huang ${ }^{1} *$ \\ Yuanyuan Mal,* \\ Chao Lv' \\ Shaolei $\mathrm{Li}^{\mathrm{I}}$ \\ Fangliang Lu' \\ Shanyuan Zhang' \\ Daisy Dandan Wang ${ }^{2}$ \\ Peter Ping Lin ${ }^{2}$ \\ Yue Yang'
}

'Key Laboratory of Carcinogenesis and Translational Research (Ministry of Education/Beijing), Department of Thoracic Surgery II, Peking University Cancer Hospital \& Institute, Beijing, People's Republic of China; ${ }^{2}$ Department of Oncology, Cytelligen, San Diego, CA, USA

*These authors contributed equally to this work
Purpose: This study aimed to explore the potential application of circulating tumor cells (CTCs) in predicting the therapeutic effect of neoadjuvant chemotherapy (NAC) in non-small -cell lung cancer (NSCLC).

Methods: Using integrated subtraction enrichment and immunostaining-fluorescence in situ hybridization, the serial CTCs of patients with NSCLC were detected in $7.5 \mathrm{~mL}$ of blood at baseline and after two cycles of cisplatin-based NAC, and all aneuploidies of chromosome 8 were examined in the enriched CTCs. Tumor responses were evaluated radiologically with serial chest computed tomography (CT) using the response evaluation criteria in solid tumors and microscopically using the tumor cell necrosis rate (TCNR) of the resected specimen after NAC. Results: After two cycles of cisplatin-based NAC, $89 \%(8 / 9)$ of the patients with radiological partial response to NAC had reduced CTC numbers, while $73 \%(8 / 11)$ of the patients with stable disease exhibited increased CTC numbers $(P=0.0098)$. On pathological examination, $90 \%(9 / 10)$ of patients with a TCNR lower than $30 \%$ had $>1$ CTC post-NAC, while $80 \%(4 / 5)$ of patients with a TCNR higher than $30 \%$ had $\leq 1$ CTC post-NAC $(P=0.017)$. In aneuploidy analysis, the positive rate $(\mathrm{CTC}>0)$ of triploid CTCs was found to have increased after NAC, in contrast with the tetraploid and multiploid CTCs. Furthermore, tetraploid and multiploid CTCs were found to be significantly downregulated in the patients with partial response to NAC.

Conclusion: The correlations of aneuploid CTCs with both radiological and pathological responses in patients with NSCLC who received NAC were summarized, and the findings indicate that enumerating and karyotyping aneuploid CTCs can serve as a surrogate marker for disease monitoring in NSCLC.

Keywords: non-small-cell lung cancer, circulating tumor cell, neoadjuvant chemotherapy, therapeutic response

\section{Introduction}

Lung cancer is the leading cause of cancer-related death worldwide. ${ }^{1}$ However, advances in therapeutic modalities have greatly improved the outcome for patients with early-stage non-small-cell lung cancer (NSCLC), and radical surgery remains the cornerstone of treatment. Systemic chemotherapy in patients with completely resected NSCLC is usually required, as postoperative cisplatin-based chemotherapy can prolong their survival. ${ }^{2,3}$ In recent years, neoadjuvant chemotherapy (NAC) has become an important therapeutic strategy in patients with non-metastatic NSCLC for its potential advantages over the adjuvant setting: ${ }^{4,5} \mathrm{NAC}$ is better tolerated than postoperative chemotherapy and provides a direct assessment of tumor response; it 
may also improve the radical resection rate and reduce the risk of downstage nodal disease, which has a clear impact on prognosis. ${ }^{6,7}$ However, poor response to NAC means delayed operation timing, which not only increases postoperative morbidity and mortality but also shows no better prognosis than surgery alone. Therefore, the effective evaluation of therapeutic response plays a critical role in guiding subsequent treatment in patients with NSCLC who undergo NAC.

Circulating tumor cells (CTCs) are shed from primary and secondary tumors that migrate into the circulatory system, and they can predict prognosis in several cancer types, including breast, colorectal, prostate, and small-cell lung cancers. ${ }^{8-10}$ The CELLSEARCH ${ }^{\circledR}$ system (Veridex, Raritan, NJ, USA) - approved by the US Food and Drug Administration - has proved a reliable and reproducible CTC enumeration technique in various cancer types ${ }^{11,12}$ and has been used by many studies that have demonstrated the prognostic significance of CTCs and found that changes in CTC count may predict chemotherapy efficacy in advanced cancers. ${ }^{13}$ However, CTC monitoring in surgically resectable NSCLC treated with NAC has not yet been fully elucidated. The reason for this lies in the low CTC detection rate described for NSCLC - representing only $21 \%$ of the total population ${ }^{14}$ by CELLSEARCH, which may be due to heterogeneity in the epithelial celladhesion molecules (EpCAMs) expressed on the cell surface of CTCs. ${ }^{15}$ However, CTCs may lose EpCAMs during epithelial-mesenchymal transition (EMT), ${ }^{16,17}$ which is a predominant mechanism for tumor cell invasion and metastasis and which occurs more commonly in NSCLC, resulting in missed detection with EpCAM-dependent strategies. In order for CTCs to be an effective surrogate response biomarker in NSCLC, an efficient and reliable detection method is required.

Chromosomal instability is a common feature in solid tumors, ${ }^{18}$ and the gain of chromosome 8 is more widely seen in aggressive lung tumors in mice. The identification of CTCs with aneuploidy in chromosome 8 may help with the evaluation of the heterogeneous phenotype of CTCs in NSCLC. ${ }^{19}$ In the present study, an EpCAM-independent method of subtraction enrichment and immunostainingfluorescence in situ hybridization (SE-iFISH) ${ }^{20}$ was used to enrich and characterize aneuploid CTCs in patients with surgically resectable NSCLC undergoing NAC. By monitoring CTCs in real-time during the process of NAC, the correlations between CTC enumeration and karyotype with radiological and pathological responses were identified and investigated.

\section{Materials and Methods}

\section{Patients and Treatment}

A total of 36 patients with histologically confirmed NSCLC who were recommended to undergo NAC at the weekly multidisciplinary team (MDT) meeting in the Thoracic Cancer Center of Peking University Cancer Hospital were enrolled between December 2014 and January 2016. Baseline resectability for each patient was confirmed by a team of thoracic surgeons at the meeting prior to the beginning of NAC.

Routine tests for staging were performed as follows: contrast chest computed tomography (CT), enhanced brain magnetic resonance imaging, abdominal ultrasonography, bone scintigraphy or positron emission tomography/computed tomography, and essential laboratory tests, including those for serum tumor markers (such as carcinoembryonic antigen [CEA]). Fibrobronchoscopic biopsy and CTguided percutaneous biopsy, respectively, were applied to central and peripheral localized tumors. Clinical staging was based on the TNM Classification of Malignant Tumors, 7th edition.

All patients were programmed to receive two cycles of cisplatin-based NAC. Patients with squamous cell carcinoma received gemcitabine $1250 \mathrm{mg} / \mathrm{m}^{2}$ on days 1 and 8 and cisplatin $75 \mathrm{mg} / \mathrm{m}^{2}$ on day 1 . Patients with adenocarcinoma received pemetrexed $500 \mathrm{mg} / \mathrm{m}^{2}$ and cisplatin $75 \mathrm{mg} / \mathrm{m}^{2}$ on day 1 . Both regimens were repeated on day 21 .

This study was approved by the Ethics Committee of Peking University Cancer Hospital and was performed according to the principles in the Declaration of Helsinki. Written informed consent, which included a statement giving approval for their disease information to be used and their blood samples to be applied for the present study, was obtained from all patients.

\section{Radiological Response}

A contrast chest CT scan was obtained within four weeks of the two NAC treatment cycles. Tumor response was determined using response evaluation criteria in solid tumors (RECIST). ${ }^{21}$ The longest dimensions of the primary tumors were measured and recorded by a team of experienced radiologists in our MDT who were blinded to the CTC detection results. After two cycles of NAC, the 
disappearance of the lesion was defined as a complete response (CR), a reduction of more than $30 \%$ in the longest dimension of the lesion was defined as a partial response (PR), and an increase of more than $20 \%$ in the longest dimension or the appearance of new lesions was defined as having progressive disease (PD). All other outcomes were defined as stable disease (SD).

\section{Pathological Response}

The medical records, including CT images, of each patient were presented at the MDT meeting after two cycles of NAC in order to decide subsequent treatment, and the resectability of each tumor was evaluated again to identify candidates for surgery Four to six weeks after the cessation of chemotherapy, surgical exploration, resection, or mediastinal lymph node dissection were conducted empirically and 22 patients received surgery. All resected specimens were sent for routine pathological analysis and examined histologically by experienced pulmonary pathologists. At least five hematoxylin and eosin-stained slides of the gross residual tumors were assessed for each patient, and the tumor cell necrosis rate (TCNR) was semi-quantitatively estimated by the percentage of the cross-sectional areas of necrosis, fibrosis, and inflammation on each slide. The results for all slides were averaged together to determine the mean values of TCNR.

\section{Detection of CTCs Using SE-iFISH}

Based on previous studies, ${ }^{22,23}$ CTC enrichment was performed using SE-iFISH. A total of $7.5 \mathrm{~mL}$ of peripheral blood was collected from each patient prior to and after the two cycles of NAC. The collected blood was centrifuged at $600 \times \mathrm{g}$ for 5 minutes, after which the sedimented cells were centrifuged at $400 \times \mathrm{g}$ for $5 \mathrm{~min}-$ utes on top of $3 \mathrm{~mL}$ of non-hematopoietic cell-separation matrix. Immunomagnetic particles conjugated with antiCD45 monoclonal antibodies were cultivated with supernatant for 10 minutes to remove the leukocytes (Cytelligen, San Diego, Ca, USA). The supernatants were collected and subjected to a magnetic separation of beads, after which the bead-free solution was spun at $500 \times \mathrm{g}$ for 2 minutes. The pellet was then thoroughly mixed with $100 \mu \mathrm{L}$ cell fixative, followed by application to the formatted and coated CTC slide (Cytelligen, San Diego, CA, USA).

Next, we performed iFISH on the resulting samples according to the kit's instructions (Cytelligen). Prepared samples on the coated slides were hybridized for $4 \mathrm{~h}$ with the Vysis Centromere Probe (CEP8) Spectrum Orange (Abbott Laboratories, Abbott Park, IL, USA), followed by an incubation with Alexa Fluor 594-conjugated monoclonal anti-CD45 antibodies (Cytelligen) at room temperature for $30 \mathrm{~min}$. Finally, we used 4-6-diamidino- 2-phenylindole (DAPI) (Life Technologies, Carlsbad, CA, USA) to stain the nuclei. CTC was defined as DAPI+, CD45- cells and identified chromosome 8 aneuploidy was found under fluorescence microscope. At least two pathologists evaluated the CTC enumeration.

\section{Statistical Analysis}

All statistical analyses were performed using SPSS software version 22.0 (SPSS, Chicago, IL, USA). The CTC enumerations in different stages were compared using the nonparametric Kruskal-Wallis test or Mann-Whitney $U$-test, while the CTC enumerations pre- and post-NAC were compared using the Wilcoxon rank-sum test. The correlations between CTC prevalence at different thresholds and clinical characteristics were compared using Fisher's exact test, as were the correlations between CTC changes and CT or pathological response. Two-tailed $P<$ 0.05 values were considered to be statistically significant.

\section{Results}

\section{Clinical Characteristics}

The clinical characteristics of the enrolled patients are given in Table 1. The study population included 28 men (78\%) and 8 women (22\%), with a median age of 60 years (39-73 years). Histological subtypes included 10 (28\%) adenocarcinomas and 26 (72\%) squamous cell carcinomas. All patients had an Eastern Cooperative Oncology Group performance status of 0 or 1 and received two complete cycles of cisplatin-based doublet NAC. CT scans were performed on all the patients after two cycles of NAC. Subsequently, 17 (47\%) of enrolled patients underwent surgery after NAC, while 19 (53\%) patients chose to receive chemoradiotherapy.

\section{CTC Distribution at Baseline}

Based on the presence of aneuploidy, a common feature of cancer cells, CTCs were identified by detecting ploidy in chromosome 8 using SE-iFISH. Prior to NAC, $7.5 \mathrm{~mL}$ of blood was collected from each patient, with $30(83.3 \%)$ samples having CTC counts $\geq 1$ (range, 1-51). The positive CTC rates were then analyzed at three different thresholds (CTC count $>0,>5$, and $>10$ ), and their 
Table I Patient Demographics

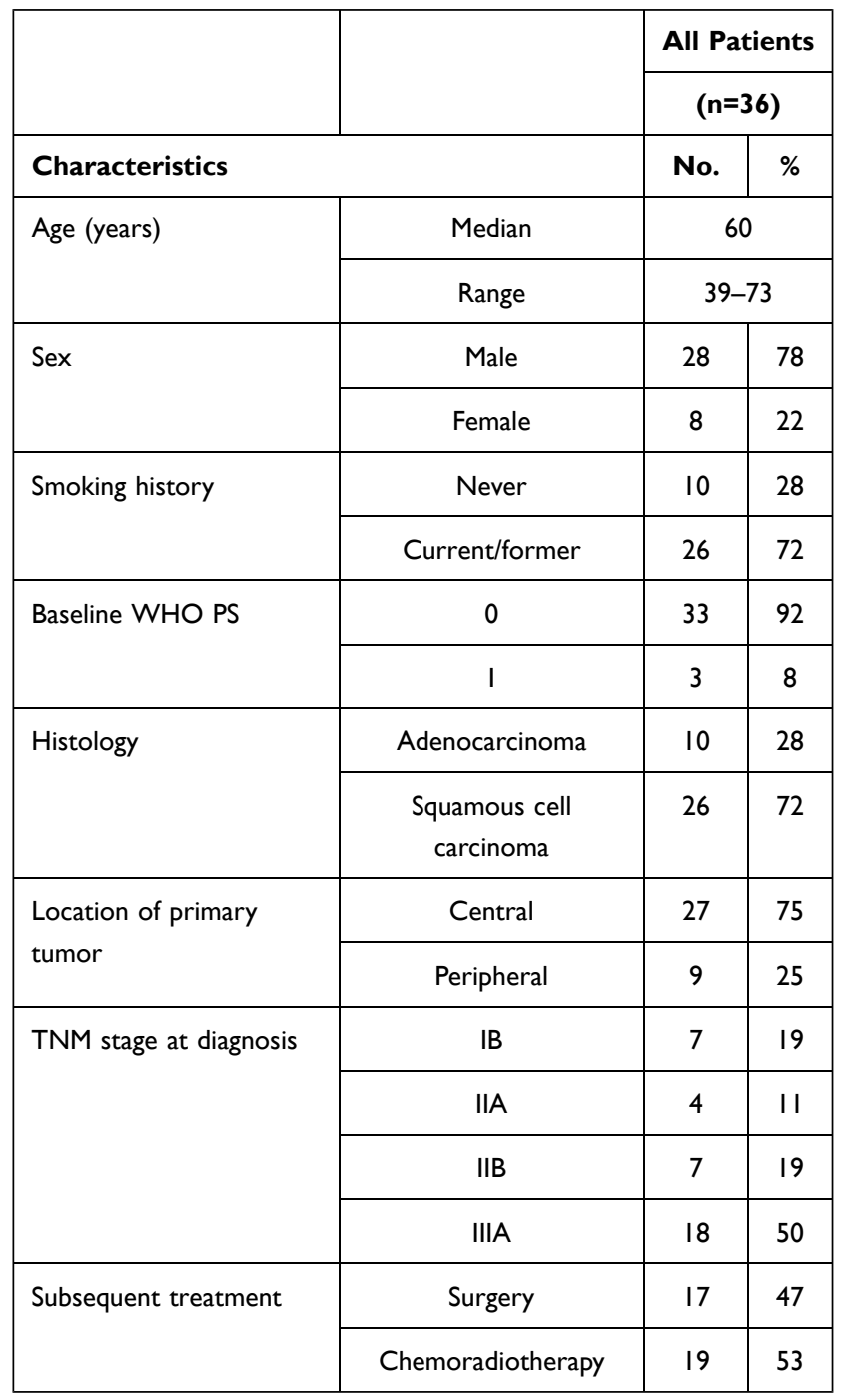

associations with the clinical characteristics for these samples were assessed (see Table 2). No significant differences in CTC detection rate were found between different groups of age, sex, histology, smoking history, tumor location, and CEA serum marker. As expected, however, significant correlations were observed between higher positive CTC rates and higher TNM stages or lymph-node metastasis at the three different thresholds.

Additionally, the number of CTCs in stage IB patients was $0.6 \pm 0.8$ (mean $\pm \mathrm{SD}$ ), the number in stage II patients was $6.5 \pm 6.0$, and the number in stage IIIA patients was $14.6 \pm 14.0$, the differences between which were significantly different ( $P=0.001$; see Figure 1A). Higher numbers of CTCs were observed in patients with lymph-node metastasis (N1-2) compared with patients with N0 diseases $(P<0.0001$; see Figure 1B).

\section{CTC Variation Associated with the} Therapeutic Efficacy of NAC

On the basis of the RECIST criteria, radiological evaluation was performed after two cycles of NAC, and PR occurred in 13 patients $(36 \%)$, SD in 19 patients $(53 \%)$, and $\mathrm{PD}$ in 4 patients $(6 \%)$. Twenty-two patients that include $10 \mathrm{PR}$ and $12 \mathrm{SD}$ had undergone subsequent surgery and their blood samples were collected for CTC evaluation before surgery. The number of CTCs increased after NAC, but no significant difference was seen $(P=$ 0.627 ; see Figure 2A).

The groups of patients with PR and those with SD were then compared. A significant decline in the number of CTCs after NAC $(P=0.013)$ was seen in the PR group, while a significant increase in the number of CTCs after NAC $(P=0.045)$ was seen in the SD group (see Figure 2B). Excluding two cases (1 PR and $1 \mathrm{SD}$ ) with no detectable CTC at both time points, 8 out of $9 \mathrm{PR}$ patients exhibited decreased CTC numbers after NAC and 8 out of 11 SD patients exhibited increased CTC numbers after NAC $(P=0.01$; see Figure $2 \mathrm{C})$, showing concordance with radiological response by chest $\mathrm{CT}$ evaluation.

The pathological responses of 15 out of the 22 patients who received radical resection after NAC were obtained. Five of the patients had a response with a TNCR of more than $30 \%$, which was empirically defined as pathological partial response (pPR). Nine out of ten $(90 \%)$ patients who failed to reach $\mathrm{pPR}$ had CTC counts $>1$ after NAC, while four out of the five $(80 \%)$ patients who reached $\mathrm{pPR}$ had CTC counts $\leq 1$ after NAC $(P=0.017$; see Figure 2D), indicating that the presence of aneuploid CTCs after NAC could predict pathological response in patients with NSCLC.

\section{Analysis of Different Karyotypes in Aneuploidy CTCs}

Heterogeneous polysomic chromosome 8 was observed in the CTCs; images of monoploidy, triploidy, tetraploidy, and multiploidy of chromosome 8 in the CTCs are shown in Figure 3A. The variations of these aneuploid CTCs were analyzed in all 36 blood samples taken from patients before NAC and the 22 samples taken from the patients who underwent radical resection after NAC. The positive rate $(\mathrm{CTC}>0)$ of triploid CTCs increased from $66.7 \%$ before NAC to $72.7 \%$ after NAC, whereas the positive rates of tetraploid and multiploid CTCs decreased 
Table 2 CTC Prevalence at Baseline and Association with Clinical Characteristics $(n=36)$

\begin{tabular}{|c|c|c|c|c|}
\hline & & \multicolumn{3}{|c|}{ Patients with CTCs Above Threshold } \\
\hline & & & n (\%) & \\
\hline \multicolumn{2}{|l|}{ Characteristic } & $>0$ & $>5$ & $>10$ \\
\hline \multicolumn{2}{|l|}{ All patient $(n=36)$} & $30(83.3 \%)$ & $19(52.8 \%)$ & II (23.8\%) \\
\hline \multirow[t]{3}{*}{ Age (years) } & $\leq 60(n=18)$ & 14 (77.8\%) & $9(50.0 \%)$ & $5(27.8 \%)$ \\
\hline & $>60(n=18)$ & $16(88.9 \%)$ & $10(55.6 \%)$ & $6(33.3 \%)$ \\
\hline & $p$ value & 0.658 & $>0.99$ & $>0.99$ \\
\hline \multirow[t]{3}{*}{ Gender } & Female $(n=8)$ & $6(75.0 \%)$ & $4(50.0 \%)$ & $3(37.5 \%)$ \\
\hline & Male $(n=28)$ & $24(85.7 \%)$ & 15 (53.5\%) & $8(28.6 \%)$ \\
\hline & $p$ value & 0.596 & $>0.99$ & 0.678 \\
\hline \multirow[t]{4}{*}{ Stage } & IB $(n=7)$ & $3(42.9 \%)$ & $0(0.0 \%)$ & $0(0.0 \%)$ \\
\hline & II $(n=I I)$ & $10(90.9 \%)$ & $6(54.5 \%)$ & $2(18.2 \%)$ \\
\hline & IIIA $(n=18)$ & 17 (94.4\%) & $13(72.2 \%)$ & $9(50.0 \%)$ \\
\hline & $p$ value & 0.006 & 0.005 & 0.029 \\
\hline \multirow[t]{3}{*}{ Histology } & $\operatorname{ADC}(n=10)$ & $8(80.0 \%)$ & $5(45.0 \%)$ & $3(30.0 \%)$ \\
\hline & $\operatorname{SCC}(n=26)$ & 22 (84.6\%) & 14 (53.8\%) & $8(30.8 \%)$ \\
\hline & $p$ value & $>0.99$ & $>0.99$ & $>0.99$ \\
\hline \multirow[t]{3}{*}{ Smoking history } & Never $(n=10)$ & $8(80.0 \%)$ & $6(60.0 \%)$ & $4(40.0 \%)$ \\
\hline & Former $(n=26)$ & 22 (84.6\%) & $13(50.0 \%)$ & 7 (26.9\%) \\
\hline & $p$ value & $>0.99$ & 0.717 & 0.454 \\
\hline \multirow[t]{3}{*}{ Location } & Central $(n=27)$ & 22 (8I.5\%) & 12 (44.4\%) & $8(29.6 \%)$ \\
\hline & Peripheral $(n=9)$ & $8(88.9 \%)$ & 7 (77.8\%) & $3(33.3 \%)$ \\
\hline & $p$ value & $>0.99$ & 0.128 & $>0.99$ \\
\hline \multirow[t]{4}{*}{ T stage } & $I(n=5)$ & $5(100.0 \%)$ & 5 (100.0\%) & $4(80.0 \%)$ \\
\hline & $2(n=21)$ & 17 (8I.0\%) & 9 (42.9\%) & 5 (23.8\%) \\
\hline & $3(n=10)$ & $8(80.0 \%)$ & $5(50.0 \%)$ & $2(20.0 \%)$ \\
\hline & $p$ value & 0.558 & 0.069 & 0.034 \\
\hline \multirow[t]{3}{*}{$\mathrm{N}$ stage } & $0(n=15)$ & $10(66.7 \%)$ & $3(20.0 \%)$ & I (6.7\%) \\
\hline & $I-2(n=2 I)$ & 20 (95.2\%) & $16(76.2 \%)$ & 10 (47.6\%) \\
\hline & $p$ value & 0.023 & 0.002 & 0.011 \\
\hline \multirow[t]{3}{*}{ CEA } & Normal $(n=25)$ & 21 (84.0\%) & $13(52.0 \%)$ & $8(32.0 \%)$ \\
\hline & Increased $(n=11)$ & $9(8 \mathrm{I} .8 \%)$ & $6(54.5 \%)$ & $3(27.3 \%)$ \\
\hline & $p$ value & $>0.99$ & $>0.99$ & $>0.99$ \\
\hline
\end{tabular}


A

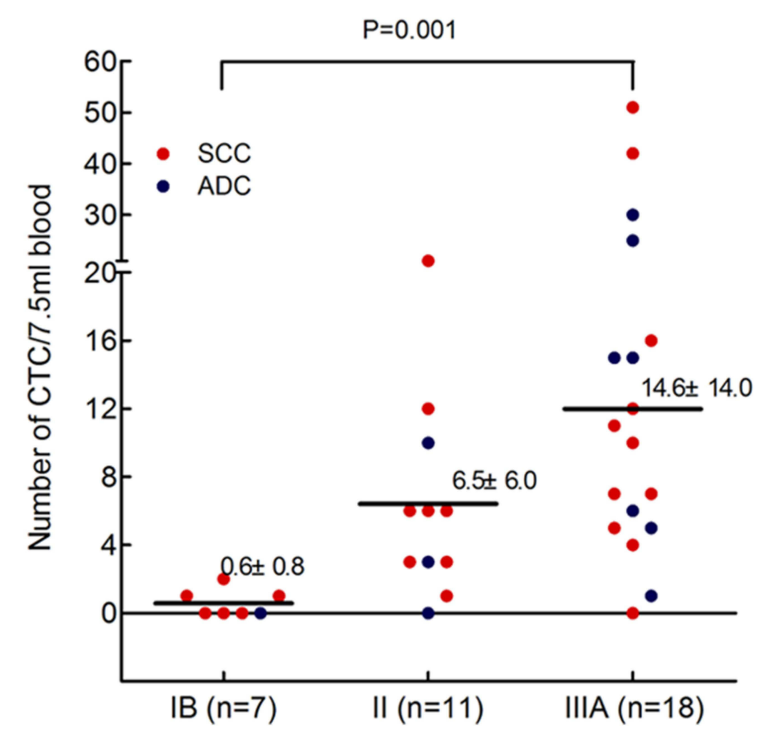

B

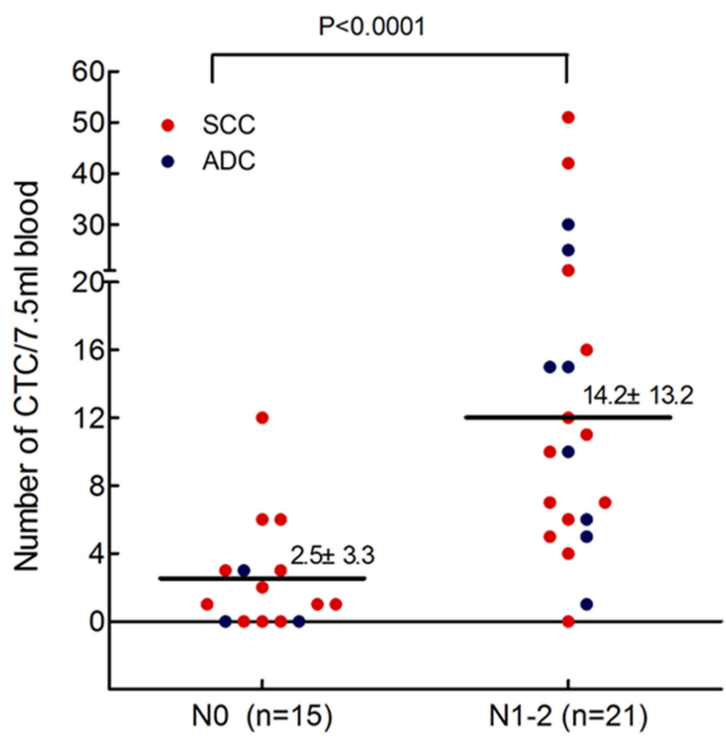

Figure I Distribution of CTC counts at baseline. Higher numbers of CTC were detected in patients with higher TNM stages $((\mathbf{A})$ Kruskal Wallis test, $p=0.00 \mathrm{I})$ and in patients with lymph node metastasis $((\mathbf{B})$ Mann-Whitney U-test, $\mathrm{p}<0.000 \mathrm{I})$.

from $52.8 \%$ and $75.0 \%$, respectively, before NAC to $36.4 \%$ and 59.1\%, respectively, after NAC (see Figure 3B), indicating that triploid chromosome 8 CTCs might be not as sensitive as tetraploid and multiploid CTCs to cisplatin-based chemotherapy. In addition, enumerations of triploid, tetraploid, and multiploid CTCs increased from $3.0 \pm 2.9,1.6 \pm 2.6$, and $5.7 \pm 8.7$, respectively, before NAC to $4.6 \pm 5.9,2.9 \pm 5.4$, and $8.4 \pm 11.6$, respectively, after NAC (see Figure 4A).

The variations of different aneuploidies before and after NAC were also evaluated in the PR and SD groups. Six out of nine $(66.67 \%)$ PR patients exhibited decreased triploid CTCs after NAC, while 8 out of 11 (72.73\%) SD patients exhibited increased triploid CTCs after NAC $(P=$ 0.175; see Figure 4B). Furthermore, in the PR group, significant downregulation of tetraploidy (all patients, $100 \%$ ) and multiploidy (8/9 patients, $88.89 \%$ ) was found, while in the SD group, distinct upregulation of tetraploidy (6/11 patients, 55\%) and multiploidy (7/11 patients, 64\%) was found $(P=0.014$ and $P=0.028$, respectively; see Figures $4 \mathrm{C}$ and D). Alterations of tetraploidy and multiploidy CTCs after chemotherapy were significantly associated with the therapeutic efficacy of NAC.

\section{Discussion}

CTCs have been extensively studied as prognostic, predictive, and dynamic biomarkers in many types of cancer. $^{24-26}$ The present study explored the significance of CTC monitoring during the course of NAC in patients with surgically resectable NSCLC using a novel EpCAMindependent SE-iFISH method to detect nonhematopoietic heteroploid CTCs.

The CELLSEARCH system, which utilizes EpCAMcoated magnetic beads to isolate CTCs, is one of the most well-known CTC enumeration technologies. A previous study using CELLSEARCH reported that there were greater numbers of CTCs in metastatic patients $(P<$ 0.001 ) than in patients in earlier stages of cancer, ${ }^{27}$ although no significant differences in CTC counts between stages I, II, and III were found and the positive rate with $\mathrm{CTC}>0$ was only $18.8 \%$ in 96 stage I-III patients. The low positivity of this EpCAM-dependent CTC detection strategy has great limitation for further study due to the heterogeneous expression of EpCAMs in solid tumors and potential EpCAM loss during the course of EMT. ${ }^{28}$ Hofman et al used isolation by size of epithelial tumor, a size-based filtration technology, to isolate the CTCs of 208 patients with NSCLC and obtained a $50 \%$ CTC detection rate by morphological examination. ${ }^{29}$ A cut-off value of $>50$ CTCs corresponded with shorter progression-free survival and overall survival, but no direct correlation was found between the number of CTCs and the disease stage. 
A

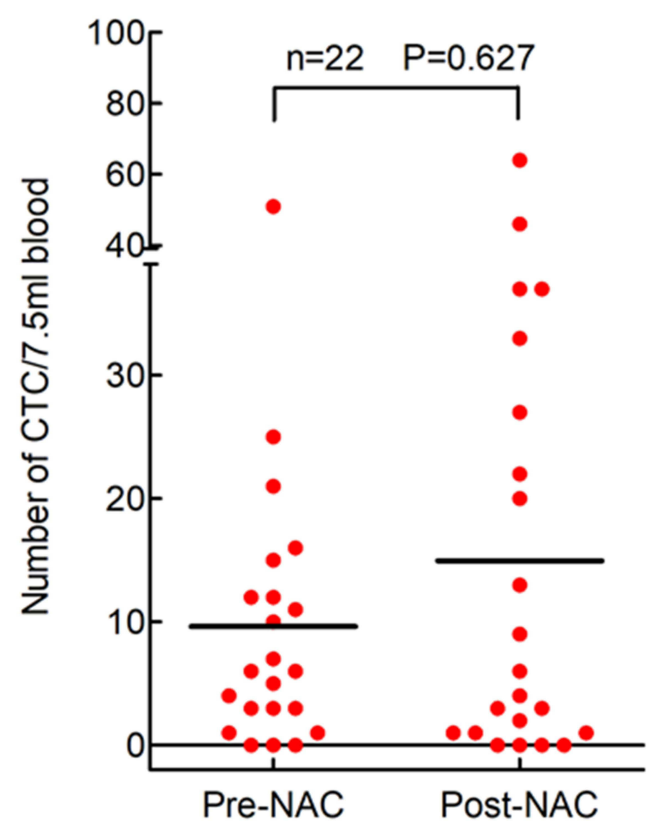

B

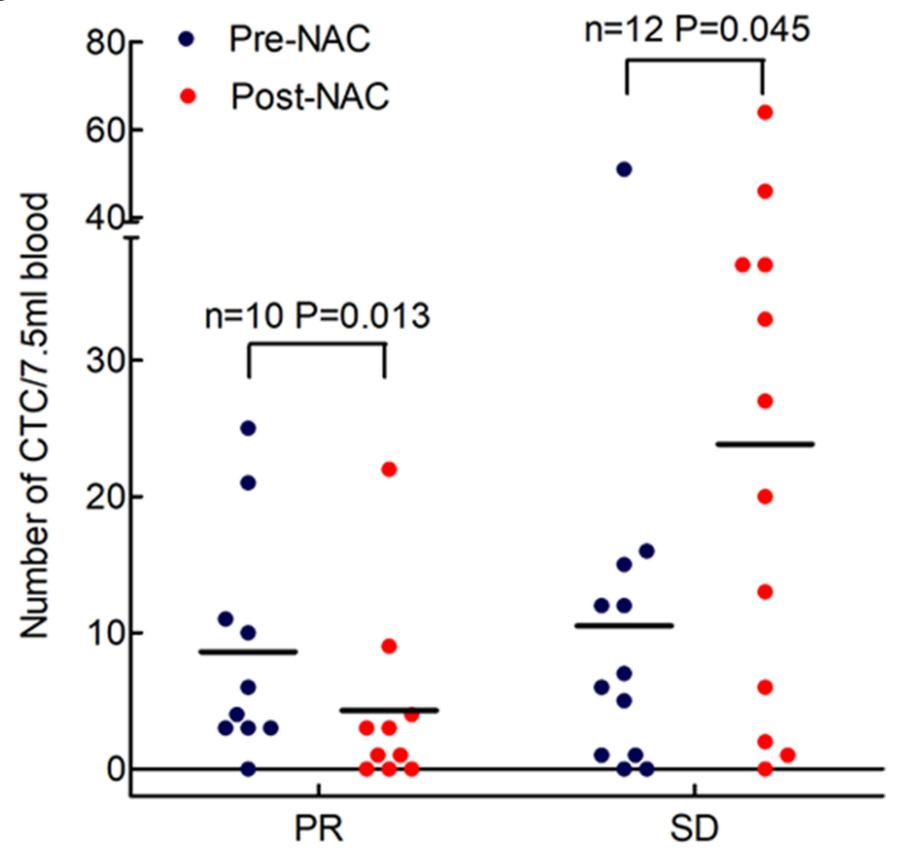

C

\begin{tabular}{|c|c|c|}
\hline NAC ORR & $\downarrow$ pre-post & $\uparrow$ pre-post \\
\hline PR & $8(88.89 \%)$ & $1(11.11 \%)$ \\
\hline SD & $3(27.27 \%)$ & $8(72.73 \%)$ \\
\hline P-value & \multicolumn{2}{|c|}{0.01} \\
\hline
\end{tabular}

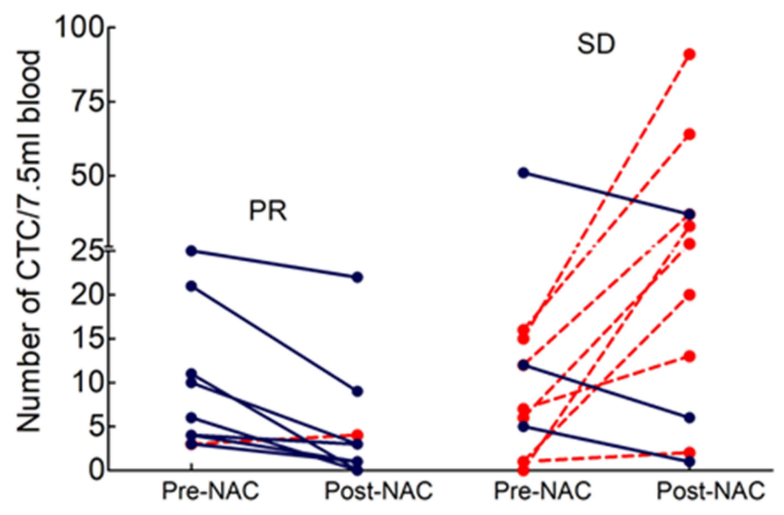

D

\begin{tabular}{|c|c|c|}
\hline TCNR & $\leq 1$ CTC & $>1$ CTC \\
\hline$\leq 30 \%$ & $1(10 \%)$ & $9(90 \%)$ \\
\hline$>30 \%$ & $4(80 \%)$ & $1(20 \%)$ \\
\hline P-value & \multicolumn{3}{|c|}{0.017} \\
\hline
\end{tabular}

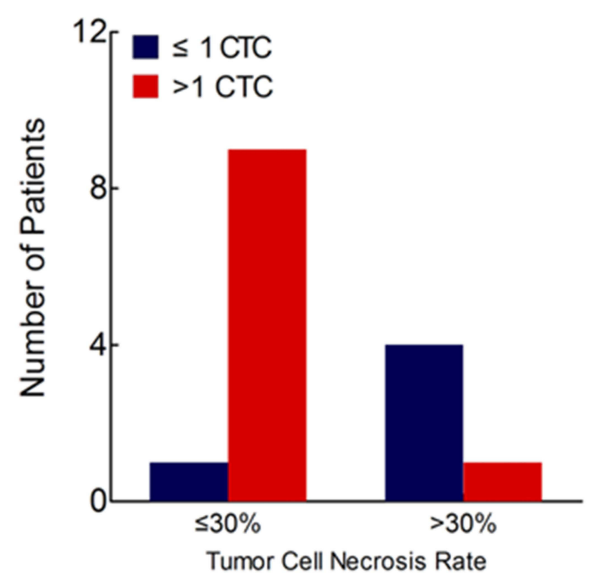

Figure 2 Changes of CTC counts before and after NAC and their correlation with therapeutic efficacy of NAC. (A) A slightly increased number of CTCs after NAC ( $\mathrm{n}=22$, Wilcoxon test, $p=0.627)$. (B) In the number of CTCs a significant decrease in PR patients $(n=10$, Wilcoxon test $p=0.013)$ and a significant increase in $S D$ patients $(n=12$, Wilcoxon test $p=0.045$ ) were seen after NAC. $(C)$ Changes of CTC counts after NAC were significantly correlated with radiological response by chest $C T$ evaluation ( $n=20$, Fisher's exact test, $\mathrm{p}=0.0 \mathrm{I}$ ). (D) No more than I CTC detected post-NAC indicating a better pathological response with more than $30 \%$ of tumor cell necrosis rate (TCNR) in the resected specimen $(n=15$, Fisher's exact test, $p=0.017)$. 
A

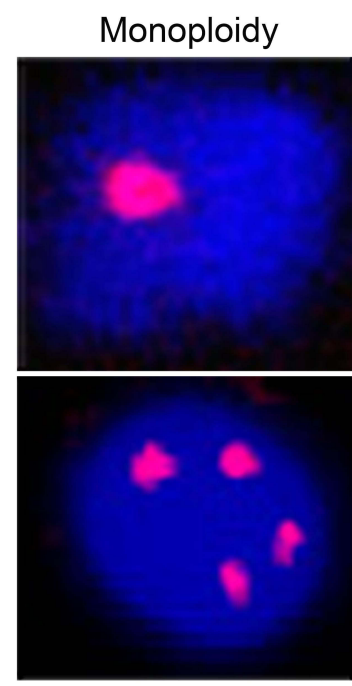

Tetraploidy

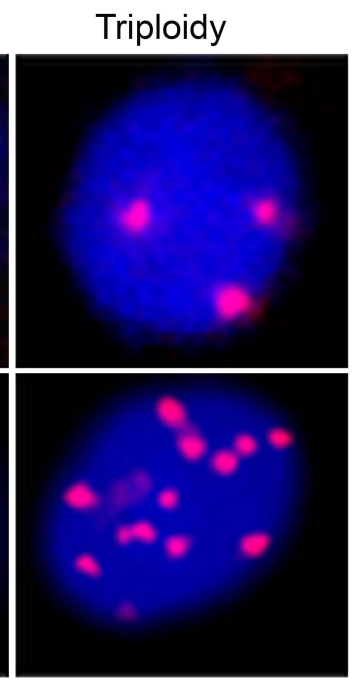

Multiploidy
B

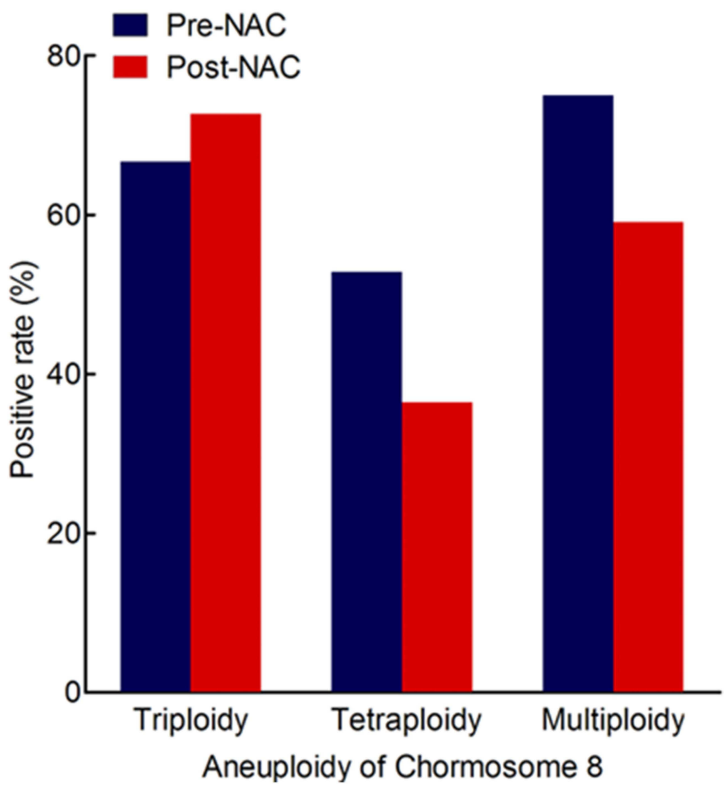

Figure 3 The number of CTCs increased progressively in radiological nonresponders during the course of 2-cycle neoadjuvant chemotherapy. (A) Representative images of monoploidy, triploidy, tetraploidy and multiploidy ( $\geq 5$ copies). Nuclei of CTCs were stained with DAPI (blue) and the chromosome 8 was detected by centromere probe 8 Spectrum Orange probe (red dots). (B) The histogram shows the increased positive rate (CTC>0) of triploid CTCs, and decreased rate of tetraploid and multiploid CTCs before $(n=36)$ and after NAC $(n=22)$.

In the present study, an EpCAM-independent approach was used to identify ploidy in CTCs using SE-iFISH to stain chromosome 8 . Of 36 patients with stage IB-IIIA NSCLC, 30 had a CTC count $\geq 1$. A previous study reported that, in advanced gastric cancer patients, SEiFISH showed a $90.5 \%$ positive rate for CTC detection compared with a detection rate of $54.8 \%$ with CELLSEARCH in the same population. ${ }^{22}$ In the present study, the CTC detection rate increased gradually from stage IB to IIIA and at different CTC thresholds $(>0,>$ 5 , and $>10)$. The present study also found that higher CTC counts were associated with higher TNM stages $(P=$ $0.0098)$ and N-stages $(P<0.0001)$, which are in turn associated with poor prognosis, indicating that TNM staging is the most effective prognostic factor for NSCLC.

One of the key features of the present study is the application of SE-iFISH in clinical practice and the exploration of the clinical significance of CTC monitoring during the process of NAC. The use of NAC and preoperative induction chemotherapy in the treatment of patients with non-metastatic NSCLC has been given an increasing amount of attention, ${ }^{6,30}$ and a recent metaanalysis of 13 randomized trials (3206 patients with NSCLC) revealed a hazard ratio for death of 0.84 (0.77$0.92, P=0.0001$ ) in favor of NAC, which is equivalent to a $6 \%$ improvement in overall survival at five years. ${ }^{31}$
However, only a small number of patients with resectable NSCLC benefit from NAC.

Radiological response based on changes in the measured dimensions of lesions identified on CT has become the standard therapeutic response assessment modality for NSCLC, although it has the limitation of simple structural imaging and there are concerns about cumulative radiation exposure. $^{32}$ In the present study, CTC counts were significantly decreased in patients who responded to NAC and increased in those who did not. CTC numbers were found to be modulated by chemotherapy intervention in 20 patients who presented positive CTCs in at least one sample, and this change predicted radiological response by CT evaluation.

Pathological response examined from a resected specimen is believed to be the most robust surrogate marker of survival for patients undergoing NAC. ${ }^{33}$ An association between pathological complete response (pCR) and survival is consistently found following NAC. ${ }^{34,35}$ Depierre reported that $11 \%$ of 179 patients with stage IB-IIIA NSCLC who were treated with NAC achieved pCR and had a 0.42 relative risk of death $(P<0.001) .{ }^{4}$ However, the RECIST criteria for CT evaluation used to determine response to NAC are not always in accordance with pathological response; this may be because CT provides only a macroscopic evaluation of the primary tumor, whereas, 
A

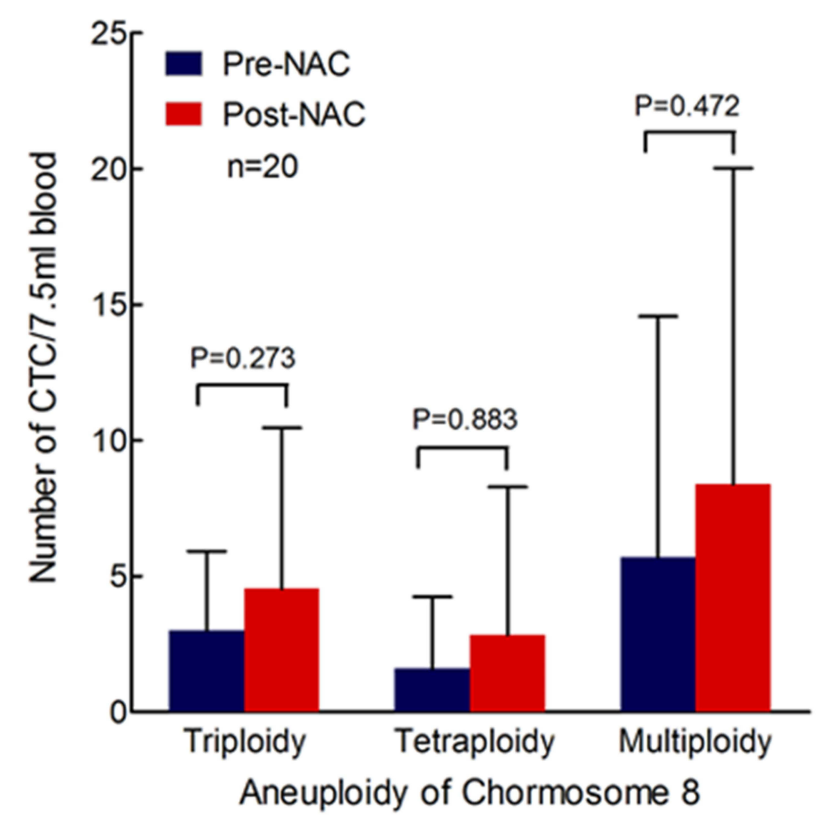

C

\begin{tabular}{|c|c|c|}
\hline Tetraploidy & \pre-post & $\uparrow$ pre-post \\
\hline PR & $9(100 \%)$ & $0(0)$ \\
\hline SD & $5(45.45 \%)$ & $6(54.55 \%)$ \\
\hline P-value & \multicolumn{2}{|c|}{0.014} \\
\hline
\end{tabular}

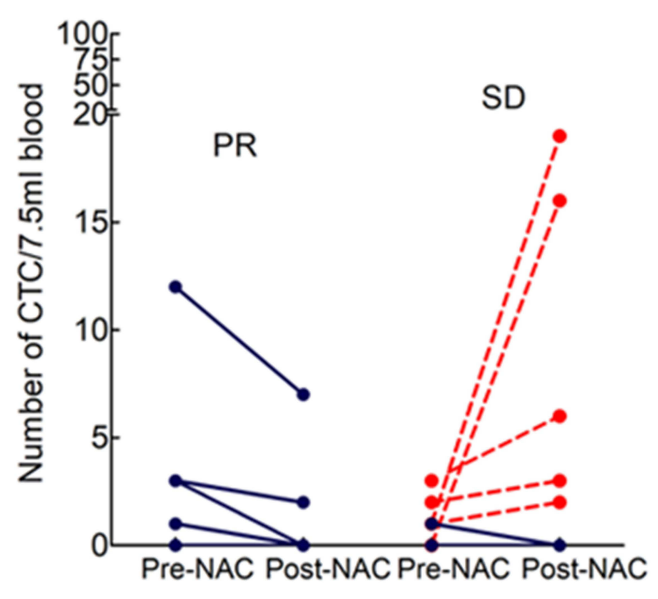

B

\begin{tabular}{|c|c|c|}
\hline Triploidy & $\downarrow$ pre-post & $\uparrow$ pre-post \\
\hline PR & $6(66.67 \%)$ & $3(33.33 \%)$ \\
\hline SD & $3(27.27 \%)$ & $8(72.73 \%)$ \\
\hline P-value & \multicolumn{2}{|c|}{0.175} \\
\hline
\end{tabular}

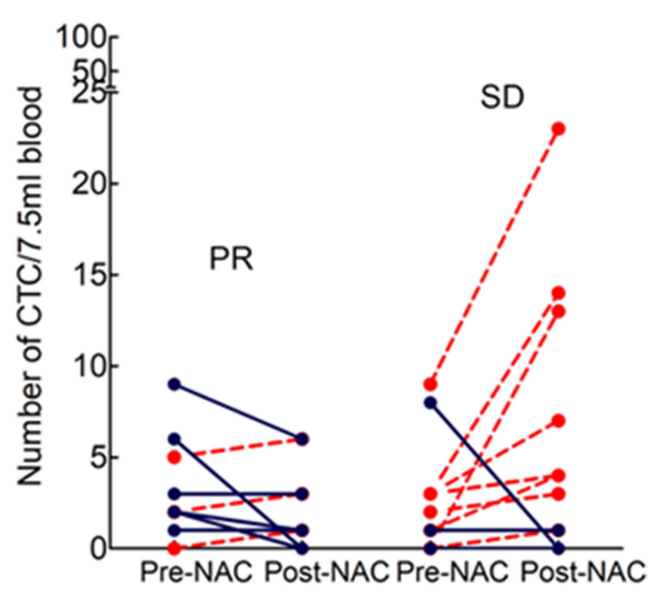

D

\begin{tabular}{|c|c|c|}
\hline Multiploidy & \pre-post & $\uparrow$ pre-post \\
\hline PR & $8(88.89 \%)$ & $1(11.11 \%)$ \\
\hline SD & $4(36.36 \%)$ & $7(63.64 \%)$ \\
\hline P-value & \multicolumn{2}{|c|}{0.028} \\
\hline
\end{tabular}

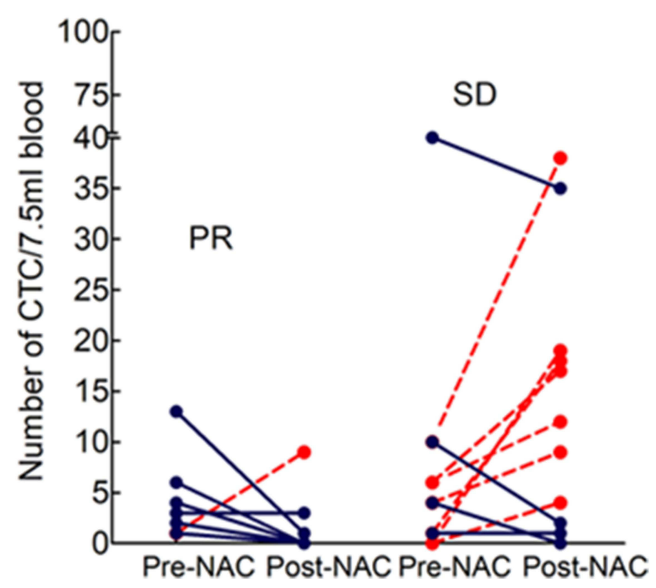

Figure 4 Variations of different aneuploid CTCs in NSCLC patients treated with NAC $(n=20)$. (A) Demonstrates slightly increased numbers of triploid, tetraploid and multiploid CTCs after NAC (Wilcoxon test $p$ value, $0.273,0.883$ and 0.472 , respectively). (B-D) shows the correlation of changes of triploid, tetraploid and multiploid CTCs with radiological response after NAC respectively (Fisher's exact test $p=0.1746,0.028,0.028$ ). 
histologically, NSCLC tumors are heterogeneous in composition and include cancer cells, stromal tissue, and associated inflammatory cells. ${ }^{36}$ In the present study, patients with a greater extent of tumor regression microscopically had fewer CTCs after treatment, while much more numbers of CTCs could be detected after NAC when tumor regression was not so obvious, indicating that the presence of CTCs after NAC was correlated with pathological response to some extent. Although the results of the present study are in the context of a small sample size, they are logical and readily comprehensible. In fact, pathological response exhibited the microscopic status of the tumors after NAC, which proved to be correlated with the number of CTCs in the peripheral blood of the patients. However, radiological assessment is based on a tumor's morphological changes before and after chemotherapy, which can also be indicated by changes in CTC counts before and after NAC.

The reliability of radiological response is increasingly being questioned as a reliable surrogate for survival in patients with surgically resectable NSCLC. In the present study, SE-iFISH-detected CTCs correlated well with both CT and pathological evaluation, making CTCs a reliable surrogate response marker in nonmetastatic NSCLC. A validated pharmacodynamic biomarker for response assessment is still needed, as it would be radiation-free and non-invasive. The results of the present study suggest that CTCs might fulfill the criteria for a surrogate response biomarker.

In the present study, heteroploid chromosome 8-identified by centromere probe-FISH-was observed in detected CTCs, and its correlation with the therapeutic response of patients with NSCLC was investigated. Aneuploidy refers to the state of a cell that contains an abnormal number of chromosomes, and it is found in the majority $(70-90 \%)$ of cancer cells. ${ }^{37}$ Despite the high frequency of its occurrence, however, the role of specific aneuploidy in disease pathogenesis is poorly understood. ${ }^{19}$ Pavelka et al designed a yeast study and found that, when under a strong selective pressure, aneuploidy could help in the generation of phenotypic variation and adaptation in yeast. $^{38}$ Another study supported the theory that widespread aneuploidy could be a positively selected driver of tumorigenesis due to its driving role in adaptive evolution by providing a fitness advantage under specific circumstances. $^{39}$ The present study identified the variation of aneuploid CTCs during the course of NAC, finding that different karyotypes of CTCs presented varying degrees of change after NAC. An elevated proportion of triploid chromosome 8 CTCs was the adaptive consequence of cisplatin-based chemotherapy, exhibiting the more obvious drug resistance of triploid CTCs compared with that of tetraploid or multiploid CTCs. Similar results have been reported by $\mathrm{Li}$ et al in a previous study of advanced gastric cancer. $^{22}$ Validation of the results consolidates the current understanding that drug resistance might be an intrinsic property of aneuploid cells. ${ }^{40}$ In addition, the present study observed a decreased number of tetraploidy and multiploidy CTCs in the patients with $\mathrm{PR}$ and an increased number of these types of CTC in the patients with SD.

One limitation of our study needs to be stated is that the detection efficiency of aneuploid CTC may be insufficient. Lerebour et $\mathrm{al}^{41}$ studied chromosome 8 abnormalities in 77 NSCLC patients and found $40 \%$ of them showed losses of hyperploidy in chromosome 8 . In our study, 2 of 22 enrolled patients had failed to detect aneuploid CTC before and after NAC, which means certain patients would be missed with the SE-iFISH technique and the potential clinical application would be limited. Another limitation is that our cohort included relatively more male and squamous cell carcinoma, which is certainly not very representative of a NSCLC cohort.

However, understanding the role of aneuploidy in lung cancer improves our knowledge of its pathogenesis and may lead to new avenues for treatment.

\section{Conclusion}

The translational research described in the present study is of high clinical value because it offers a better understanding of NAC and the way in which monitoring therapeutic effect could help improve treatment strategies. To our knowledge, this is the first study to report on the significant correlations between variations of aneuploid CTCs and radiological and pathological response after NAC in patients with non-metastatic NSCLC. The findings suggest that CTCs can serve as a surrogate marker for response evaluation of NAC in real time and as a potential intermediary end point for survival in patients with surgically resectable NSCLC. We also found that the upregulation of triploid chromosome 8 CTCs showed a weak correlation with drug resistance to cisplatin-based chemotherapy, and the downregulation of tetraploidy or multiploidy CTCs was significantly associated with NAC sensitivity.

These preliminary data are promising for the potential clinical use of CTCs, although it is generated from 
a limited sample of patients. Phenotyping and karyotyping of CTCs by SE-iFISH demonstrates tumor heterogeneity, and more observation time and follow-up studies are required to identify whether these characteristics of CTCs are predictive of survival.

\section{Acknowledgments}

We would like to acknowledge the hard and dedicated work of all the staff that implemented the intervention and evaluation components of the study.

\section{Funding}

There is no funding to report.

\section{Disclosure}

No authors have stated any conflicts of interest.

\section{References}

1. Siegel RL, Miller KD, Jemal A. Cancer statistics, 2017. CA Cancer J Clin. 2017;67(1):7-30.

2. Berghmans T, Paesmans M, Meert AP, et al. Survival improvement in resectable non-small cell lung cancer with (neo)adjuvant chemotherapy: results of a meta-analysis of the literature. Lung Cancer. 2005;49 (1):13-23. doi:10.1016/j.lungcan.2005.01.002

3. Pignon JP, Tribodet H, Scagliotti GV, et al. Lung adjuvant cisplatin evaluation: a pooled analysis by the LACE Collaborative Group. $J \quad$ Clin Oncol. 2008;26(21):3552-3559. doi:10.1200 JCO.2007.13.9030

4. Depierre A, Milleron B, Moro-Sibilot D, et al. Preoperative chemotherapy followed by surgery compared with primary surgery in resectable NSCLC. J Clin Oncol. 2001;20:247-253.

5. Paz-Ares L, Corral J. Preoperative chemotherapy for non-small-cell lung cancer: a systematic review and meta-analysis of individual participant data. Lancet. 2014;383(9928):1561-1571.

6. Scagliotti GV, Pastorino U, Vansteenkiste JF, et al. Randomized Phase III study of surgery alone or surgery plus preoperative cisplatin and gemcitabine in stages IB to IIIA non-small-cell lung cancer. J Clin Oncol. 2012;30(2):172-178. doi:10.1200/JCO.2010.33.7089

7. Burdett S, Stewart LA, Rydzewska L. A systematic review and meta-analysis of the literature: chemotherapy and surgery versus surgery alone in non-small cell lung cancer. $J$ Thorac Oncol. 2006;1:611-621.

8. Alix-Panabieres C, Pantel K. Circulating tumor cells: liquid biopsy of cancer. Clin Chem. 2013;59(1):110-118. doi:10.1373/ clinchem.2012.194258

9. Punnoose EA, Atwal S, Liu W, et al. Evaluation of circulating tumor cells and circulating tumor DNA in non-small cell lung cancer: association with clinical endpoints in a Phase II clinical trial of pertuzumab and erlotinib. Clin Cancer Res. 2012;18(8):2391-2401. doi:10.1158/1078-0432.CCR-11-3148

10. Muinelo-Romay L, Vieito M, Abalo A, et al. Evaluation of Circulating Tumor Cells and Related Events as Prognostic Factors and Surrogate Biomarkers in Advanced NSCLC Patients Receiving First-Line Systemic Treatment. Cancers. 2014;6(1):153-165. doi:10.3390/cancers6010153

11. Allard WJ, Matera J, Miller MC, et al. Tumor cells circulate in the peripheral blood of all major carcinomas but not in healthy subjects or patients with nonmalignant diseases. Clin Cancer Res. 2004;10:6897-6904. doi:10.1158/1078-0432.CCR-04-0378
12. Riethdorf S, Fritsche H, Muller V, et al. Detection of circulating tumor cells in peripheral blood of patients with metastatic breast cancer: a validation study of the CellSearch system. Clin Cancer Res. 2007;13(3):920-928. doi:10.1158/1078-0432.CCR-06-1695

13. Miller MC, Doyle GV, Terstappen LW. Significance of circulating tumor cells detected by the cellsearch system in patients with metastatic breast colorectal and prostate cancer. $J$ Oncol. 2010;2010:617421. doi:10.1155/2010/617421

14. Krebs MG, Sloane R, Priest L, et al. Evaluation and prognostic significance of circulating tumor cells in patients with non-smallcell lung cancer. $J$ Clin Oncol. 2011;29(12):1556-1563. doi: $10.1200 / J C O .2010 .28 .7045$

15. Cristofanilli M, Budd GT, Ellis MJ. Circulating tumor cells, disease progression, and survival in metastatic breast cancer. $N$ Engl $J$ Med. 2004;351(8):781-791. doi:10.1056/NEJMoa040766

16. Mikolajczyk SD, Millar LS, Tsinberg P, et al. Detection of EpCAM-Negative and Cytokeratin-Negative Circulating Tumor Cells in Peripheral Blood. J Oncol. 2011;2011:252361. doi:10.1155/ 2011/252361

17. Gires O, Stoecklein NH. Dynamic EpCAM expression on circulating and disseminating tumor cells: causes and consequences. Cell Mol Life Sci. 2014;71(22):4393-4402. doi:10.1007/s00018-014-1693-1

18. Kops GJ, Weaver BA, Cleveland DW. On the road to cancer: aneuploidy and the mitotic checkpoint. Nat Rev Cancer. 2005;5 (10):773-785. doi:10.1038/nrc1714

19. Gordon DJ, Resio B, Pellman D. Causes and consequences of aneuploidy in cancer. Nat Rev Genet. 2012;13(3):189-203. doi:10.1038/ $\operatorname{nrg} 3123$

20. C W, H H, L L, et al. Preliminary investigation of the clinical significance of detecting circulating tumor cells enriched from lung cancer patients. J Thorac Oncol. 2009;4:30-36. doi:10.1097/ JTO.0b013e3181914125

21. Eisenhauer EA, Therasse P, Bogaerts J, et al. New response evaluation criteria in solid tumours: revised RECIST guideline (version 1.1). Eur J Cancer. 2009;45(2):228-247. doi:10.1016/j.ejca.2008.10.026

22. Li Y, Zhang X, Ge S, et al. Clinical significance of phenotyping and karyotyping of circulating tumor cells in patients with advanced gastric cancer. Oncotarget. 2014;5:6594-6602. doi:10.18632/ oncotarget. 2175

23. Feng G, Zhang H, Wang DD, Linda L, Lin PP. Enhanced detection and comprehensive in situ phenotypic characterization of circulating and disseminated heteroploid epithelial and glioma tumor cells. Oncotarget. 2015;6(29):27049-27064. doi:10.18632/oncotarget.4819

24. Cohen SJ, Punt CJ, Iannotti N, et al. Relationship of circulating tumor cells to tumor response, progression-free survival, and overall survival in patients with metastatic colorectal cancer. J Clin Oncol. 2008;26(19):3213-3221. doi:10.1200/JCO.2007.15.8923

25. Cen P, Ni X, Yang J, Graham DY, Li M. Circulating tumor cells in the diagnosis and management of pancreatic cancer. Biochim Biophys Acta. 2012;1826(2):350-356.

26. Pailler E, Adam J, Barthelemy A, et al. Detection of circulating tumor cells harboring a unique ALK rearrangement in ALK-positive non-small-cell lung cancer. J Clin Oncol. 2013;31 (18):2273-2281. doi:10.1200/JCO.2012.44.5932

27. Tanaka F, Yoneda K, Kondo N, et al. Circulating tumor cell as a diagnostic marker in primary lung cancer. Clin Cancer Res. 2009;15(22):6980-6986. doi:10.1158/1078-0432.CCR-09-1095

28. Krebs MG, Hou J-M, Sloane R, et al. Analysis of circulating tumor cells in patients with non-small cell lung cancer using epithelial marker-dependent and -independent approaches. J Thorac Oncol. 2012;7:306-315. doi:10.1097/JTO.0b013e31823c5c16

29. Hofman V, Bonnetaud C, Ilie MI, et al. Preoperative circulating tumor cell detection using the isolation by size of epithelial tumor cell method for patients with lung cancer is a new prognostic biomarker. Clin Cancer Res. 2011;17(4):827-835. doi:10.1158/ 1078-0432.CCR-10-0445 
30. Decaluwe H, De Leyn P, Vansteenkiste J, et al. Surgical multimodality treatment for baseline resectable stage IIIA-N2 non-small cell lung cancer. Degree of mediastinal lymph node involvement and impact on survival. Eur $j$ Cardio-Thoracic Surg. 2009;36 (3):433-439. doi:10.1016/j.ejcts.2009.04.013

31. Song W-A, Zhou N-K, Wang W, et al. Survival Benefit of Neoadjuvant Chemotherapy in Non-small Cell Lung Cancer-An Updated Meta-Analysis of 13 Randomized Control Trials. $J$ Thorac Oncol. 2010;5:510-516. doi:10.1097/JTO.0b013e3181cd3345

32. Hicks RJ. Role of 18F-FDG PET in assessment of response in non-small cell lung cancer. $J$ Nuclear Med. 2009;50(Suppl 1):31S42S. doi:10.2967/jnumed.108.057216

33. Mouillet G, Monnet E, Milleron B, et al. Pathologic complete response to preoperative chemotherapy predicts cure in early-stage non-small-cell lung cancer. $J$ Thorac Oncol. 2012;7:841-849. doi:10.1097/JTO.0b013e31824c7d92

34. Betticher DC, Hsu Schmitz SF, Totsch M, et al. Mediastinal lymph node clearance after docetaxel-cisplatin neoadjuvant chemotherapy is prognostic of survival in patients with stage IIIA pN2 non-small-cell lung cancer: a multicenter phase II trial. J Clin Oncol. 2003;21 (9): 1752-1759. doi:10.1200/JCO.2003.11.040

35. Betticher DC, Hsu Schmitz SF, Totsch M, et al. Prognostic factors affecting long-term outcomes in patients with resected stage IIIA pN2 non-small-cell lung cancer: 5-year follow-up of a phase II study. $\mathrm{Br}$ J Cancer. 2006;94(8):1099-1106. doi:10.1038/sj.bjc.6603075
36. William N, Pataer A, Kalhor N, et al. Computed Tomography RECIST Assessment of Histopathologic Response and Prediction of Survival in Patients with Resectable Non-Small-Cell Lung Cancer after Neoadjuvant Chemotherapy. J Thorac Oncol. 2013;8:222-228. doi:10.1097/JTO.0b013e3182774108

37. Weaver BA, Cleveland DW. Does aneuploidy cause cancer? Curr Opin Cell Biol. 2006;18(6):658-667. doi:10.1016/j.ceb.2006.10.002

38. Pavelka N, Rancati G, Zhu J, et al. Aneuploidy confers quantitative proteome changes and phenotypic variation in budding yeast. Nature. 2010;468(7321):321-325. doi:10.1038/nature09529

39. Pavelka N, Rancati G, Dr Jekyll LR. Mr Hyde: role of aneuploidy in cellular adaptation and cancer. Curr Opin Cell Biol. 2010;22 (6):809-815. doi:10.1016/j.ceb.2010.06.003

40. Lee AJX, Endesfelder D, Rowan AJ, et al. Chromosomal instability confers intrinsic multidrug resistance. Cancer Res. 2011;71 (5):1858-1870. doi:10.1158/0008-5472.CAN-10-3604

41. Lerebours F, Olschwang S, Thuille B, et al. Fine deletion mapping of chromosome 8p in non-small-cell lung carcinoma. Int J Cancer. 1999;81 (6):854-858. doi:10.1002/(SICI)1097-0215(19990611)81:6<854::AIDIJC3 $>3.0 . \mathrm{CO} ; 2-1$
International Journal of General Medicine

\section{Publish your work in this journal}

The International Journal of General Medicine is an international, peer-reviewed open-access journal that focuses on general and internal medicine, pathogenesis, epidemiology, diagnosis, monitoring and treatment protocols. The journal is characterized by the rapid reporting of reviews, original research and clinical studies

\section{Dovepress}

across all disease areas. The manuscript management system is completely online and includes a very quick and fair peer-review system, which is all easy to use. Visit http://www.dovepress.com/ testimonials.php to read real quotes from published authors. 